Check for updates

Cite this: RSC Adv., 2018, 8, 23712

Received 13th May 2018

Accepted 24th June 2018

DOI: $10.1039 / c 8 \mathrm{ra04066d}$

rsc.li/rsc-advances

\section{Superhydrophobic coatings on gelatin-based films: fabrication, characterization and cytotoxicity studies $\uparrow$}

\author{
Yu Chen, (D) C Weipeng Lu, ${ }^{\text {*ac }}$ Yanchuan Guo, ${ }^{\text {*abc }}$ Yi Zhu, $^{\mathrm{c}} \mathrm{Haojun} \mathrm{Lu}^{\mathrm{c}}$ and Yuxiao $\mathrm{Wu}^{\mathrm{c}}$
}

As a degradable natural biomaterial, gelatin has good biocompatibility and nontoxicity, but gelatin is easily soluble in water which has limited its application. In order to solve this tough defect, superhydrophobic gelatin films (GSF) were prepared by first grafting silica nanoparticles onto gelatin films and then modifying silica nanoparticles with a fluorosilane coupling agent (FAS). Fourier transform infrared spectroscopy (FTIR), scanning electron microscopy (SEM), a particle size analyzer, a contact angle instrument (CA), X-ray photoelectron spectroscopy (XPS), a universal materials tester and an Incucyte ${ }^{\mathrm{TM}}$ Zoom system were used to characterize the morphology, molecular interactions, superhydrophobic performance, and cytotoxicity. Results show that $\mathrm{GSF}_{300}$ modified by silica nanoparticles with a particle size of $303 \mathrm{~nm}$ has the largest contact angle $\left(158.6^{\circ}\right.$ ). At the same time, the contact angle is still more than $150^{\circ}$ after 48 hours of infiltration in water. These results indicate that $\mathrm{GSF}_{300}$ has strong long-term water resistance. In addition, $\mathrm{GSF}_{300}$ has good mechanical strength, durability and nontoxicity. Therefore, such a durable, robust and superhydrophobic film has good potential applications in various functional biomedical aspects.

\section{Introduction}

The remarkable improvement of modern medicine requires the performance of biomedical materials to be better and has a multi-functional development trend. Among these materials, natural degradable materials have good biocompatibility and low toxicity, ${ }^{1}$ and can be absorbed or excreted by organisms to relieve patients from the pain of secondary surgery. Therefore, more and more attention has been paid to the research of natural degradable materials, and it has become a hot spot of current research. Gelatin, as a natural degradable material, is obtained by partial hydrolysis of collagen, and is a polypeptide molecular polymer composed of different amino acids. ${ }^{2}$ Owing to its excellent biological properties and nontoxicity, it is widely used in biomedical products such as biofilm materials, ${ }^{3}$ tissue engineering scaffolds, ${ }^{4}$ medical sponges, ${ }^{5}$ and capsules. ${ }^{6}$ But it's worth noting that gelatin is easily soluble in water, which seriously limits its practical application.

Due to the easy water absorption in wet environment, gelatin needs to have strong water resistance and

${ }^{a}$ Key Laboratory of Photochemical Conversion and Optoelectronic Material, Technical Institute of Physics and Chemistry, Chinese Academy of Sciences, Beijing 100190, China

${ }^{b}$ University of Chinese Academy of Sciences, Beijing 100049, China

${ }^{c}$ Hangzhou Research Institute of Technical Institute of Physics and Chemistry, Chinese Academy of Sciences, Hangzhou 310018, China. E-mail: luweipeng@mail.ipc.ac.cn; Tel: +86-571-87853765

$\dagger$ Electronic supplementary information (ESI) available. See DOI: 10.1039/c8ra04066d superhydrophobicity. In order to solve this intractable problem, researchers have made a lot of attempts, such as blending, grafting. For example, S. Afshar et al. developed a hydrophobic biodegradable nanocomposite gelatin films by blending starch and graphene oxide, and the static contact angle was $112.04^{\circ} \pm 4.85$, which was $40^{\circ}$ greater than pure gelatin film. ${ }^{7} \mathrm{C}$. Shi et al. prepared a new gelatin film modified by starch-based ester Starch-EDTAD-NHS (SEN), and the contact angle was found to increase from $77.8^{\circ}$ to $126.7^{\circ}$ with increasing concentration of SEN. ${ }^{8}$ Besides, gelatin/zein nanofiber films by hybrid electrospinning were reported by Deng et al., and when the weight ratio was $1: 1$, the contact angle reached the $118^{\circ}$, resulting in a good solvent resistance against water. ${ }^{9}$ From the above description, we can find that these methods can improve the hydrophobicity of gelatin to some extent. However, no reports have yet provided superhydrophobic gelatin films. In addition, it is important to note that gelatin, as a protein macromolecule, has many hydrophilic groups on the surface, such as amino and hydroxyl. So, simple blending or grafting can hardly guarantee the hydrophobic properties for a long time.

Inspired with nature surface, such as lotus leaves, water strider legs, and cicada wings, ${ }^{\mathbf{1 0}, 11}$ superhydrophobic surfaces are characterized by a static water contact angle (WCA) above $150^{\circ}$ and a sliding angle (SA) less than $10^{\circ}$, by definition. ${ }^{12,13}$ Due to the unique water-repellent property, superhydrophobic materials have gained tremendous interest, which has widespread applications in self-cleaning, ${ }^{\mathbf{1 4}, 15}$ anti-icing, ${ }^{16}$ metal anti-corrosion, ${ }^{17}$ separation of oil and water, ${ }^{18}$ especially in biomedical fields such as anti-sticking 
of protein, cells, and/or bacteria, ${ }^{19-21}$ and drug release. ${ }^{22,23}$ Therefore, the study of superhydrophobic gelatin materials is of great application value.

Herein, in order to realize the superhydrophobicity of gelatin films, suitable amount of formaldehyde and glycerol were firstly added to the film as crosslinkers and plasticizers respectively to improve its mechanical properties. Then we prepared silica nanoparticles with different particle sizes by Stöber $^{24}$ sol-gel process. Next, the obtained silica nanoparticles were deposited on the modified gelatin film to improve the surface roughness, further functionalized with fluoroalkylsilane (FAS) in an ethanolic solution to obtain superhydrophobic gelatin film. As far as we know, this was the first time to prepare superhydrophobic gelatin film material. More importantly, the super hydrophobic gelatin films have good mechanical strength, durability and nontoxicity, which greatly expands the biological application of superhydrophobic material. The results are summarized and discussed in the following sections.

\section{Experimental}

\subsection{Materials}

Gelatin (obtained from bones, with an approximate molecular weight of 100000 , viscosity value of $4.9 \mathrm{mPa}$ s) was provided from Baotou Dongbao Bio-Tech Co., Ltd. (Baotou, China). Formaldehyde solution (37-40 wt\%), glycerol, tetraethoxysilane (TEOS), ammonia solution (28 wt\%), acetic acid, and ethanol absolute were purchased from Sinopharm Chemical Reagent Co., Ltd. (Shanghai, China). 1H,1H,2H,2H-Perfluorodecyltrimethoxysilane (FAS) was obtained from Shangfluoro Co., Ltd. (Shanghai, China). The human fibroblast cell lines (hFOB 1.19) were purchased from Cell bank of Chinese academy of sciences (Shanghai, China). All chemical reagents were analytical grade and deionized water with a resistivity of $18.2 \mathrm{M} \Omega \mathrm{cm}$ was used in all of experiments.

\subsection{Preparation of gelatin film modified with formaldehyde and glycerol (GE)}

For the preparation of GE, $0.8 \mathrm{~g}$ of gelatin was added into $25 \mathrm{~mL}$ deionized water with stirring at $45{ }^{\circ} \mathrm{C}$ until dissolved completely, followed by the addition of $0.14 \mathrm{~g}$ glycerol as plasticizer and $15 \mu \mathrm{L}$ formaldehyde solution as cross-link agent and continued mixing at $45^{\circ} \mathrm{C}$ for $30 \mathrm{~min}$ with constant stirring. The completely solubilized solution was cast evenly on a leveled polytetrafluoroethylene dish $(80 \mathrm{~cm} \times 80 \mathrm{~cm})$ and put an oven at $40{ }^{\circ} \mathrm{C}$ until the films dried. The completely dried films were peeled off from plates and preconditioned in a constant temperature and humidity chamber controlled at $45{ }^{\circ} \mathrm{C}$ and $50 \% \mathrm{RH}$ at least for $72 \mathrm{~h}$ before further test and use. The pure gelatin film was also prepared by the above method as control.

\subsection{Synthesis of silica nanoparticles}

For synthesis four different sizes nanoparticles $(100-400 \mathrm{~nm})$, silica nanoparticles were prepared with the procedure originally described by Stöber et al. i.e., hydrolysis of tetraethyl orthosilicate in an ethanol solution containing water and ammonia. In a typical experiment, tetraethyl orthosilicate was mixed with ethanol and deionized water, and then ammonia solution was added drop by drop to the mixed solution. The solution was stirred intensively at room temperature for $8 \mathrm{~h}$. The experimental details are presented in Table 1. After the reaction, the silica nanoparticles were collected by centrifugation and washed with ethanol and water in sequence, and finally dispersed in ethanol for further experiments.

\subsection{Preparation of GE films modified with silica nanoparticles (GS)}

The prepared silica nanoparticles were ultrasonically dispersed in ethanol (2.5\%) for 30 minutes. Then the obtained GE $(2 \mathrm{~cm} \times$ $2 \mathrm{~cm}$ ) were immersed in the suspension for $10 \mathrm{~h}$. Next, the films were removed from the solution at a withdrawal speed of 50 $\mathrm{mm} \mathrm{min}^{-1}$ and dried at room temperature. Finally, the obtained films were preconditioned in a constant temperature and humidity chamber controlled at $45{ }^{\circ} \mathrm{C}$ and $50 \% \mathrm{RH}$ at least for $48 \mathrm{~h}$ before further test and use. $\mathrm{GS}_{100}, \mathrm{GS}_{200}, \mathrm{GS}_{300}$ and $\mathrm{GS}_{400}$, represent the films that are modified by the nanoparticles of $127,225,303$ and $447 \mathrm{~nm}$, respectively.

\subsection{Preparation of GS films modified with FAS (GSF)}

Firstly, the ethanol solution of FAS with a volume fraction of $1 \%$ was prepared and the $\mathrm{pH}$ value of the solution was adjusted to 4.0 by using glacial acetic acid. Next, the GS were immersed in the obtained solution for 24 hours at room temperature. Finally, the products after FAS coating were subsequently washed with ethanol to remove excess reactants and then dried in oven for $12 \mathrm{~h}$ at $45{ }^{\circ} \mathrm{C} . \mathrm{GSF}_{100}, \mathrm{GSF}_{200}, \mathrm{GSF}_{300}$ and $\mathrm{GSF}_{400}$, respectively represent the films $\mathrm{GS}_{100}, \mathrm{GS}_{200}, \mathrm{GS}_{300}$ and $\mathrm{GS}_{400}$ that are modified with the FAS.

\subsection{Mechanical durability of $\mathrm{GSF}_{300}$}

Abrasion and peeling tests were employed to evaluate mechanical stability of $\mathrm{GSF}_{300}$. For the abrasion tests, a $500 \mathrm{~g}$ weight was put on the $\mathrm{GSF}_{300}$ to provide a pressure of $5 \mathrm{kPa}$, and then the balance weight moved forward from the left to right for $10 \mathrm{~cm}$ in one direction with the surface abraded by the sandpaper. This process was defined as an abrasion cycle. WCAs were measured after every abrasion cycle. For the tape peeling test, the $\mathrm{GSF}_{300}$ was brought in contact with the surface of the $3 \mathrm{M}$ adhesive tape, and then the adhesive tape was peeled off from the films. This process was repeated several times. In each cycle, a new piece of adhesive tape was used.

Table 1 Chemicals for the preparation of silica nanoparticles

$\mathrm{NH}_{3} \cdot \mathrm{H}_{2} \mathrm{O}$ /TEOS (volume ratio), with constant volume ratio of

\begin{tabular}{lll} 
Sample & $\mathrm{C}_{2} \mathrm{H}_{5} \mathrm{OH} / \mathrm{TEOS}=33$ & Diameter/nm \\
\hline
\end{tabular}

$\begin{array}{lll}\mathrm{S} 1 & 1.3 & 127 \\ \mathrm{~S} 2 & 1.5 & 225 \\ \mathrm{~S} 3 & 2.0 & 303 \\ \mathrm{~S} 4 & 2.7 & 447\end{array}$




\subsection{Cell culture and proliferation}

Cell culture and proliferation were assessed by human fibroblast cell lines (hFOB 1.19). Cells were cultured in Dulbecco's Modified Eagle's Medium/F-12 (DME/F-12) supplemented with $10 \%$ fetal bovine serum. All media contained $100 \mathrm{U} \mathrm{mL}^{-1}$ of

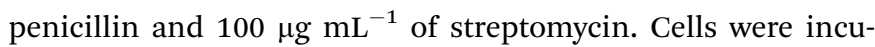
bated at $37{ }^{\circ} \mathrm{C}$ and supplemented with $5 \% \mathrm{CO}_{2}$ in the humidified chamber. Test on extraction was carried out in accordance with the instruction of ISO 10993-12: 2002. The extraction media of the pure gelatin and GSF were prepared using serum free cell culture medium (DMEM), with the extraction ratio (the ratio of specimen surface area to extraction medium) of $3 \mathrm{~cm}^{2}$ $\mathrm{mL}^{-1}$, and then incubated in a humidified atmosphere with $5 \%$ $\mathrm{CO}_{2}$ at $37{ }^{\circ} \mathrm{C}$ for $24 \mathrm{~h}$. The extraction medium was stored at $4{ }^{\circ} \mathrm{C}$ before the further test. Cell culture medium (DMEM) was used as a negative control.

\subsection{Characterization}

2.8.1 Field emission scanning electron microscope (FESEM). Microstructure of the film surface was obtained by FESEM (vltra55, Carl Zeiss SMT Pte Ltd.). Samples were sprayed with gold coating and observed under an accelerating voltage of $3 \mathrm{kV}$. Images were registered at 20000 and 60000 of magnifications.

2.8.2 Particle size measurements. The particle size in solution was analyzed by the dynamic light scattering (DLS) method (litesizer ${ }^{\mathrm{TM}}$ 500, Anton Paar $\mathrm{GmbH}$ ). The silica nanoparticles were dispersed in ethanol.

2.8.3 FTIR analysis. FTIR absorbance spectra was recorded on a spectrometer (Bruker Tensor II, Germany) using the digital attenuated total reflectance (ATR) accessory. Samples were scanned from 4000 to $400 \mathrm{~cm}^{-1}$. Each spectrum obtained at a resolution of $0.3 \mathrm{~cm}^{-1}$ was an average of 32 consecutive scans.

2.8.4 Contact angle measurements. The water contact angle (WCA) and sliding angle (SA) were measured by optical contact angle measuring instrument (Dataphysics, Germany) with a $5 \mu \mathrm{L}$ deionized water droplet at ambient temperature. The results were measured four times on different positions of the sample surface.

2.8.5 Mechanical properties. Film thickness was reported as the mean of the measurements at four different points on each specimen taken with a micrometer (Dongguan, China). Tensile strength and percent elongation of films were measured by using a texture analyzer (Instron, America) after equilibration at $50 \% \mathrm{RH}$ for $72 \mathrm{~h}$. The analysis was performed on $10 \mathrm{~mm} \times$ $20 \mathrm{~mm}$ strips. The initial grip separation and crosshead speed were set at $12 \mathrm{~mm}$ and $50 \mathrm{~mm} \mathrm{~min}^{-1}$, respectively. Percentage elongation $(E \%)$ and tensile strength of the film were calculated with following equations:

$$
E \%=\frac{l-l_{0}}{l} \times 100 \%
$$

Tensile strength $=\frac{\text { maximum force }(\mathrm{N})}{\text { thickness }(\mathrm{mm}) \times \text { width }(\mathrm{mm})} \times 100 \%$ $l_{0}$ represents the initial length of the film, $l$ represents the length of the film at the break point. The results were the average measurements on five samples.

2.8.6 X-ray photoelectron spectroscopy. The compositions of film surface were detected by a PHI VersaProbe X-ray photoelectron spectroscopy (XPS) in an ultrahigh vacuum chamber $\left(1 \times 10^{6} \mathrm{mmHg}\right)$. The energy pass was $100.0 \mathrm{eV}$, and the energy step was set at $1.0 \mathrm{eV}$.

2.8.7 Long time dynamic cell imaging and functional analysis. The effect of the extraction on hFOB 1.19 cells was observed in real time using an Incucyte ${ }^{\mathrm{TM}}$ Zoom system (EssenBio, USA). The extraction was placed in a 96-multi well plate and cultured with a 2000 cell per well supplemented with $10 \%$ FBS and $50 \mathrm{mg} \mathrm{mL}^{-1}$ ascorbic acid at $37^{\circ} \mathrm{C}$ in a $5 \% \mathrm{CO}_{2}$ for 7 days. Phase contrast images were obtained every three hours to provide data for confluence determination.

\section{Results and discussion}

\subsection{Formation of the interactions between gelatin, silica nanoparticles and FAS}

The possible model for the reaction between gelatin, silica nanoparticles and FAS is showed in Scheme 1. As we mentioned above, gelatin is comprised of a variety of amino acids, such as alanine, arginine, phenylalanine, proline and 4-hydroxyproline.

During the preparation of gelatin films, most hydrophilic groups such as amino group and carboxyl group move inward and form hydrogen bonds with internal hydrophilic groups, and at this point, the surface of the films are some hydrophobic groups, such as aliphatic chain and aryl groups. ${ }^{25}$ It is worth noting that the carbonyl group is generally on the hydrophobic side due to trans structure of the peptide bond. When silica nanoparticles are deposited on the surface of the film, it's very likely that a large number of hydroxyl groups on the silica nanoparticles can form abundant hydrogen bonds with the carbonyl group on the surface of the gelatin with hydroxyl groups acting as $\mathrm{H}$ donors and carbonyl groups acting as $\mathrm{H}$ acceptors. Next, due to the hydrolysis of the $\mathrm{Si}-\mathrm{OC}_{2} \mathrm{H}_{5}$ of FAS in acid condition, the obtained hydrolyzed fluorosilane can condense with hydroxyl groups of silica nanoparticles. Finally, the superhydrophobic gelatin-based films (GSF) are formed by above process.

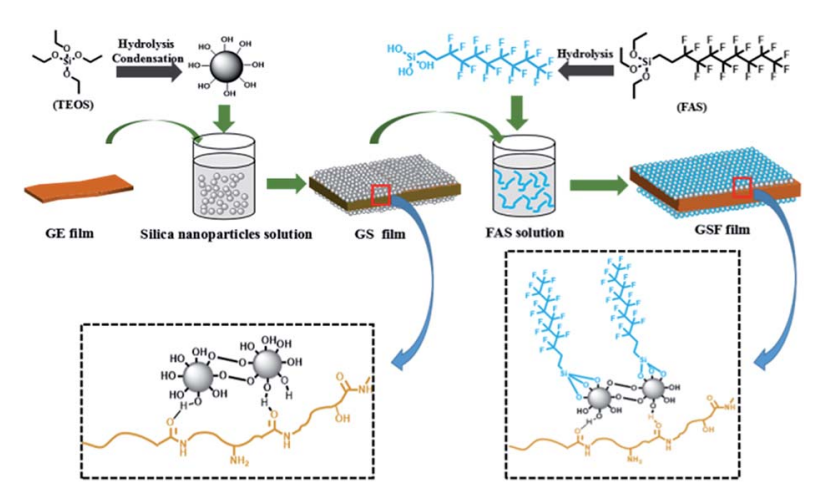

Scheme 1 Schematic illustration of the fabrication of superhydrophobic films (GSF). 

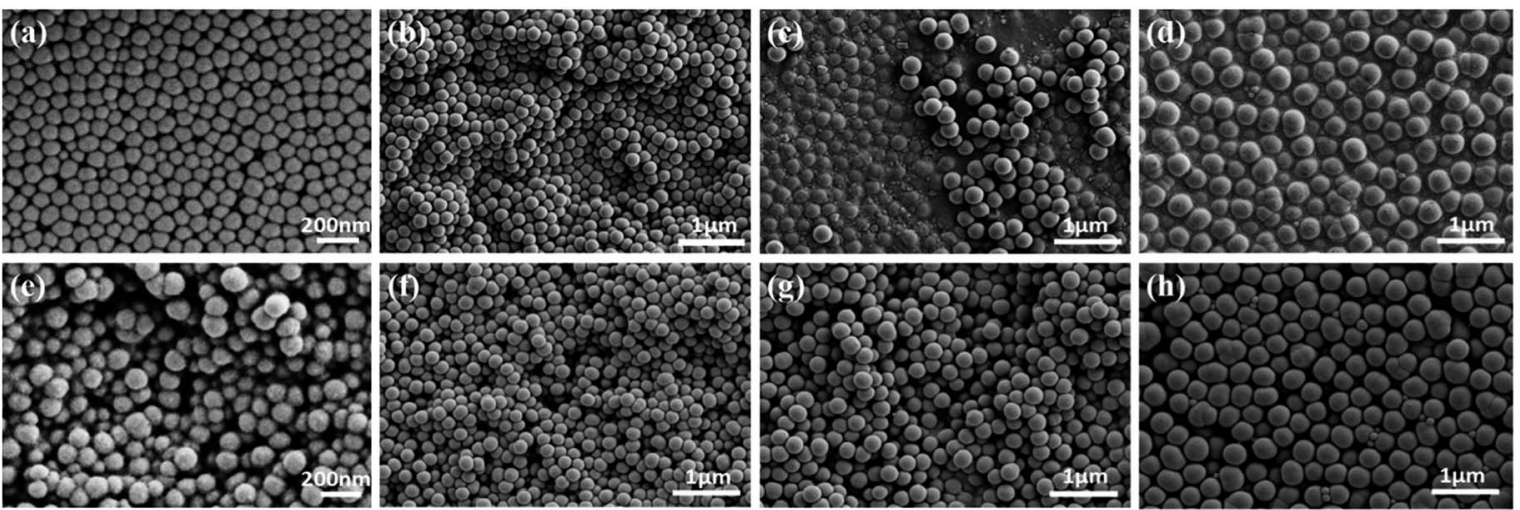

Fig. 1 FESEM top-view images of various films. (a) GS $\mathrm{S}_{100}$, (b) GS $\mathrm{GS}_{200}$, (c) GS 300 , (d) GS 400 , (e) GSF 100 , (f) GSF 200 , (g) GSF 300 , and (h) GSF 400 .

\subsection{Surface analysis of GS and GSF}

Table 1 provides different $\mathrm{NH}_{3} \mathrm{H}_{2} \mathrm{O}$ /TEOS volume ratios for the preparation of silica nanoparticles with a constant $\mathrm{C}_{2} \mathrm{H}_{5} \mathrm{OH} /$ TEOS volume ratio of 33 via standard Stöber process. It indicates that the sizes of silica nanoparticles increase from 100 to $400 \mathrm{~nm}$ with the increase in the volume ratio of $\mathrm{NH}_{3} \mathrm{H}_{2} \mathrm{O}$ /TEOS from 1.3 to 2.7. After weighing and calculation, the GE/silica nanoparticles/FAS ratio of GSF is about $3: 1: 0.2$ (wt/wt). In addition, the DLS measurements show that average hydrodynamic diameters of the silica nanoparticles is $127,225,303$ and $447 \mathrm{~nm}$, and the polydispersity index is $0.031,0.138,0.049$ and 0.001 respectively (Fig. S1, ESI $\dagger$ ), which demonstates that the four kinds of silica nanoparticles have good homogeneous size distribution. The morphology of GS and GSF was characterized by the FESEM. Fig. 1a-h shows the top-view images of $\mathrm{GS}_{100}$, $\mathrm{GS}_{200}, \mathrm{GS}_{300}, \mathrm{GS}_{400}, \mathrm{GSF}_{100}, \mathrm{GSF}_{200}, \mathrm{GSF}_{300}$, and $\mathrm{GSF}_{400}$, respectively. It indicates that the sizes of silica nanoparticles are basically consistent before and after being modified by FAS coupling agent. The images also demonstrate that silica nanoparticles are dispersed evenly on the films and have a tendency to be agglomerated to form clusters, which resulted in a rough morphology.

FTIR spectras of pure gelatin film, GE, $\mathrm{GS}_{300}$ and $\mathrm{GSF}_{300}$ are showed in Fig. 2a-d respectively. In Fig. 2a, the spectrum of pure gelatin depicts characteristic peaks at $1629 \mathrm{~cm}^{-1}$, $1531 \mathrm{~cm}^{-1}$ and $1237 \mathrm{~cm}^{-1}$, which corresponds to amide I, amide II and amide III respectively. The amide I band is dominantly attributed to the $\mathrm{C}=\mathrm{O}$ stretching vibrations. The amide II and III bands are due to the $\mathrm{N}-\mathrm{H}$ bending vibrations and $\mathrm{C}-\mathrm{N}$ stretching vibrations respectively. ${ }^{26}$ As shown in Fig. 2b, the peak situates around $1037 \mathrm{~cm}^{-1}$ might be related to glycerol. $^{27,28}$ Besides, the characteristic peak positions at $1630 \mathrm{~cm}^{-1}, 1533 \mathrm{~cm}^{-1}$ and $1238 \mathrm{~cm}^{-1}$ are shifted slightly higher compared with the pure gelatin, which may be caused by the occurrence of the Schiff base reactions between the $-\mathrm{NH}_{2}$ of the gelatin and Formaldehyde. ${ }^{29,30}$ The spectrum in Fig. 2c shows three significant peaks of silica nanoparticles. The strongest peak is at $1068 \mathrm{~cm}^{-1}$, which is due to the asymmetric stretching vibration of $\mathrm{Si}-\mathrm{O}-\mathrm{Si}$ bonds in the silica nanoparticles. Another peak appearing at $792 \mathrm{~cm}^{-1}$ is associated with the bending

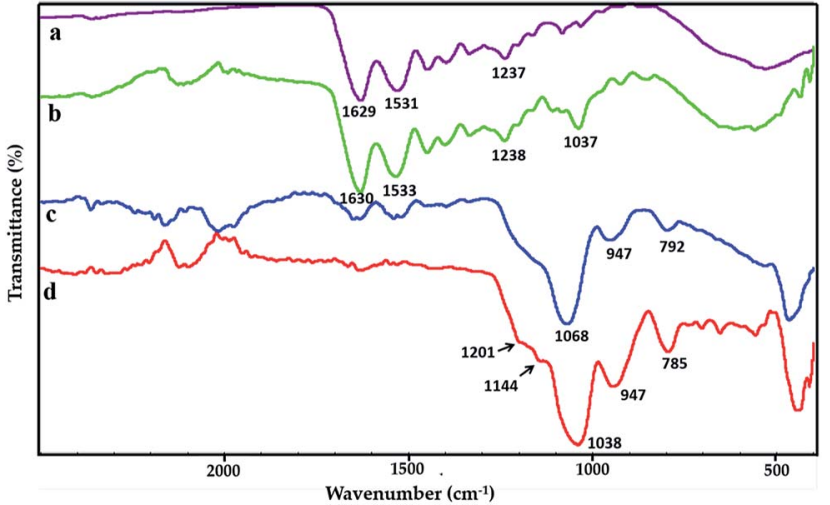

Fig. 2 FTIR spectra of films. (a) Pure gelatin film, (b) GE, (c) $\mathrm{GS}_{300}$, (d) $\mathrm{GSF}_{300}$

mode of $\mathrm{Si}-\mathrm{O}-\mathrm{Si}$ bonds. In addition, a weak absorption peak appears at $950 \mathrm{~cm}^{-1}$, which is caused by the presence of $\mathrm{Si}-\mathrm{OH}$ bonds. In the Fig. 2d, two tiny peaks at $1201 \mathrm{~cm}^{-1}$ and $1144 \mathrm{~cm}^{-1}$ superimposed on the left shoulder of the $\mathrm{Si}-\mathrm{O}-\mathrm{Si}$ peak at $1038 \mathrm{~cm}^{-1}$ are due to the stretching vibration of $\mathrm{C}-\mathrm{F}$ bonds and $\mathrm{C}-\mathrm{Si}-\mathrm{O}$ bond that arises the link between FAS molecules and the silica nanoparticles. ${ }^{31}$

In addition, XPS was selected to further explore the atomic composition of the film surface. As shown in Fig. $3 a$ and $b$, the pure gelatin film and the GE have the same three peaks at $288 \mathrm{eV}, 400 \mathrm{eV}$ and $532 \mathrm{eV}$, which can be confirmed to belong to C1 s, N1 s and O1 s respectively. And this is consistent with their compositions. In the Fig. 3c, the significant decrease of the C1 s peak at $288 \mathrm{eV}$ and almost disappearance of the $\mathrm{N} 1 \mathrm{~s}$ peak at $400 \mathrm{eV}$ are both caused by the silica nanoparticles coated on the $\mathrm{GS}_{300}$. Furthermore, the characteristic peaks of Si2 p at $103 \mathrm{eV}$ and $\mathrm{Si} 2 \mathrm{~s}$ at $154 \mathrm{eV}$ are also detected. Next, the Fig. $3 \mathrm{~d}$ shows that the $\mathrm{C} 1 \mathrm{~s}$ peak at $285 \mathrm{eV}, 291 \mathrm{eV}$ and $293 \mathrm{eV}$ are undoubted ascribed to $\mathrm{CH}_{2}, \mathrm{CF}_{2}$ and $\mathrm{CF}_{3}$ functional groups, respectively. In addition, the peaks at $688 \mathrm{eV}, 834 \mathrm{eV}$ and $862 \mathrm{eV}$ are also observed on the surface of $\mathrm{GSF}_{300}$, which can be determined to be caused by $\mathrm{CF}_{2}, \mathrm{CF}_{3}$ of FAS. So XPS characterization confirms that the silica nanoparticles and FAS molecules are successfully bonded to the gelatin film, which is consistent with the result of FTIR spectra. 


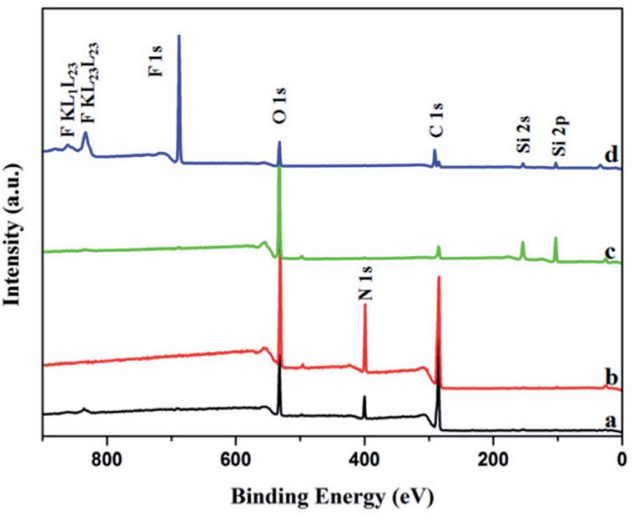

Fig. 3 XPS spectra of the surface of the films. (a) Pure gelatin film, (b) $\mathrm{GE}$, (c) $\mathrm{GS}_{300}$, (d) $\mathrm{GSF}_{300}$.

As an extremely hydrophilic substance, gelatin has abundant hydrophilic groups. But as shown in Fig. 4a, the instantaneous WCA is $102.4^{\circ} \pm 1.2$, showing a certain hydrophobicity. As we mentioned above, during the preparation of gelatin films, most hydrophilic groups move inward and form hydrogen bonds with internal hydrophilic groups, and the surface of the films is some hydrophobic groups. Therefore, the instantaneous WCA of the gelatin film is greater than $90^{\circ}$ and exhibits a certain hydrophobicity. But pure gelatin film has strong water absorbability and can absorb water after a few seconds. The WCA of GE is $113^{\circ} \pm 0.9$, which is $10^{\circ}$ larger than the pure gelatin film. This is due to the crosslinking effect of the formaldehyde and the amino group of the gelatin molecule, thus the hydrophobicity of the film is improved. Meanwhile, the Fig. $4 \mathrm{a}$ also shows that the WCAs of the films $\mathrm{GS}_{100}, \mathrm{GS}_{200}, \mathrm{GS}_{300}$ and $\mathrm{GS}_{400}$ are $18.4^{\circ} \pm 1.3$, $15.4^{\circ} \pm 1.0,14.3^{\circ} \pm 1.1$ and $17.6^{\circ} \pm 1.0$, respectively. This is attributed to the presence of a large number of hydroxyl groups on the surface of the silica nanoparticles, so the water droplets can be completely spread over the films. ${ }^{32}$ The WCAs of the films $\mathrm{GSF}_{100}, \mathrm{GSF}_{200}, \mathrm{GSF}_{300}$ and $\mathrm{GSF}_{400}$ are found to be $152.3^{\circ} \pm 0.1$, $156.6^{\circ} \pm 1.8,158.6^{\circ} \pm 1.2$ and $151.9^{\circ} \pm 1.0$, respectively. It can be seen that all the GSF exhibit high water repellency. As shown in Fig. 4b, the superhydrophobicity of GSF was further characterized in regards to the hysteresis of water contact angle which was measured by SA. The SAs of the films GSF ${ }_{100}, \mathrm{GSF}_{200}, \mathrm{GSF}_{300}$ and $\mathrm{GSF}_{400}$ are $10.8^{\circ} \pm 0.5,6.3^{\circ} \pm 0.8,5.6^{\circ} \pm 0.5$ and $9.0^{\circ} \pm 0.9$, respectively. The above results show that the WCA of GSF is more than $150^{\circ}$ and the SA is less than $10^{\circ}$, which accords with the definition of superhydrophobicity. In addition, Fig. $4 \mathrm{~b}$
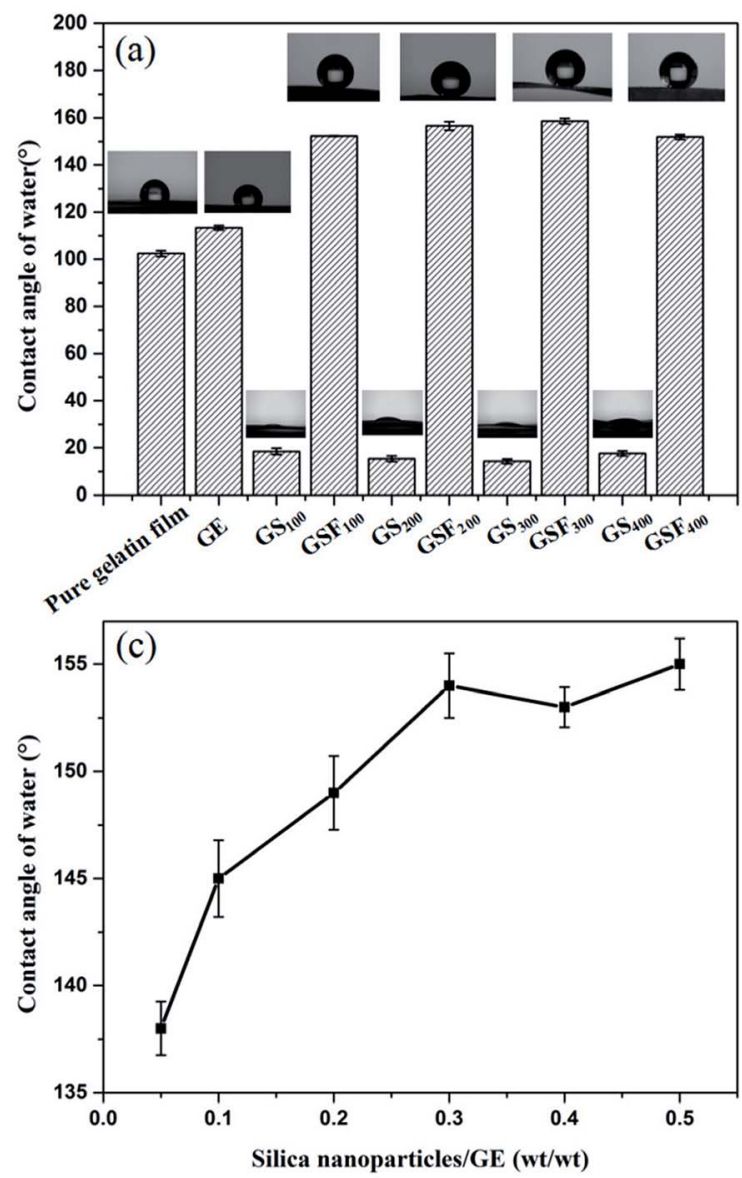
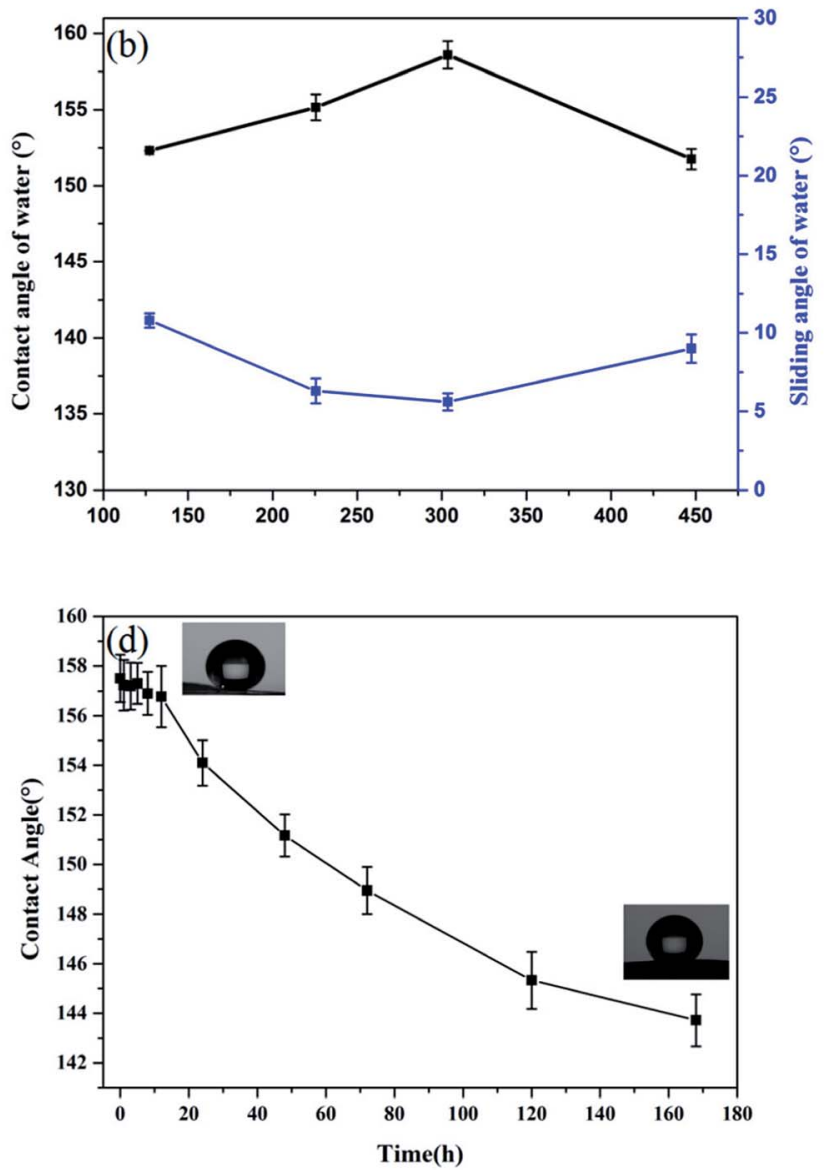

Fig. 4 (a) The WCAs on the pure gelatin, GE, GS, GSF. (b) The WCAs on the GSF modified with fluorinated silica nanoparticles of varying sizes. (c) Variation of WCA with the mass ratio of silica nanoparticles and GE. (d) The WCAs change with the soaking time in water. 
Table 2 Mechanical properties of pure gelatin, GE, GS 300 and $\mathrm{GSF}_{300}$

\begin{tabular}{lcrr}
\hline & Thickness/mm & Tensile strength/MPa & Elongation/\% \\
\hline Pure gelatin film & $0.22 \pm 0.01$ & $55.60 \pm 0.09$ & $1.99 \pm 0.09$ \\
GE & $0.20 \pm 0.02$ & $60.38 \pm 4.94$ & $5.04 \pm 0.45$ \\
GS $_{300}$ & $0.19 \pm 0.04$ & $110.01 \pm 7.02$ & $3.78 \pm 0.67$ \\
GSF $_{300}$ & $0.19 \pm 0.01$ & $83.96 \pm 12.0$ & $4.42 \pm 0.09$
\end{tabular}

indicates that the WCAs increase from $152.3^{\circ} \pm 0.1$ to $158.6^{\circ} \pm$ 1.2 by increasing the size of the silica nanoparticles modified with FAS from 127 to $303 \mathrm{~nm}$. However, as the size increases further to $447 \mathrm{~nm}$, the water contact angle decreases to $151.9^{\circ}$. We find that the obtained results are similar with the observations by Brassard et al. ${ }^{31}$ In Brassard et al. studies, the sizes of nanoparticles $(40-300 \mathrm{~nm})$ are related to roughness. As the particle size increases, the surface roughness increases, which leads to the increase of contact angle. Then we also tested the relationship between the amount of silica nanoparticles loaded and the WCA. From Fig. 4c, we can see that the WCA increases with the increase of the amount of silicon nanoparticles, when the mass ratio of silica nanoparticles to GE reaches 0.3 , the WCA is more than $150^{\circ}$, which shows superhydrophobic property. This can be explained that there is a correlation between the roughness and the silica nanoparticles as well as the WCA and roughness, that is, with the increase of the loading amount of silica nanoparticles, the roughness of the film increases continuously, which leads to the increase of WCA. When the silica nanoparticles/GE ratio of GSF is about 0.3 , the roughness of the film reaches the peak value, so, when the mass ratio of silicon nanoparticles to GE is greater than 0.3 , the roughness of the film will not continue to increase, resulting in no significant change in WCA. Therefore, 0.3 is the best silica nanoparticles/ GE ratio for preparing superhydrophobic GSF. Next, we took the $\mathrm{GSF}_{300}$ as an example to test the water resistance by immersing film into water. From Fig. $4 \mathrm{~d}$, it can be seen that after 7 day of immersing, the contact angle of the $\mathrm{GSF}_{300}$ decreases from $158.6^{\circ} \pm 1.2$ to $143.7^{\circ} \pm 1.1$, and still remains excellent water resistance.
Table 2 exhibits tensile strength, and elongation at break of the pure gelatin, GE, $\mathrm{GS}_{300}$ and $\mathrm{GSF}_{300}$. The tensile strength of GE increases by $8 \%$ compared to the pure gelatin film. This behavior can be assigned to the crosslinking effect between the aldehyde group and the amino group of the gelatin molecule. ${ }^{33}$ What's more, due to the formation of a dense and stable coating on the surface of the gelatin film by silicon nanoparticles, the tensile strength of $\mathrm{GS}_{300}$ and $\mathrm{GSF}_{300}$ increases by $98 \%$ and $51 \%$, respectively. Meanwhile, the elongation at break of $\mathrm{GE}, \mathrm{GS}_{300}$ and $\mathrm{GSF}_{300}$ increased by $153 \%$, $90 \%$ and $122 \%$, respectively compared to the pure gelatin film. This finding can be explained by the possibility that glycerol molecules can form hydrogen bonds with gelatin molecules, which can destroy the hydrogen bonds between and within the molecular chains of gelatin, weaken the interaction within the gelatin molecules, enhance the elasticity and increase the elongation at break. ${ }^{34,35}$ Based on above datas, it demonstates that the $\mathrm{GSF}_{300}$ has good mechanical properties. Furthermore, it is worth noting that after 7 days of immersion in water, the $\mathrm{GSF}_{300}$ remains in its original shape, but some swelling occurs. The tensile strength, and elongation at break is $18.24 \pm 8.35$ and $40.18 \pm 7.44$, respectively. Besides, to prove that the GSF has a mechanical durable superhydrophobic surface, abrasion and peeling tests of $\mathrm{GSF}_{300}$ were carried out. Fig. 5a shows the plot of the WCA of superhydrophobic paper as a function of abration times, and it indicates that the WCA decreases to $150^{\circ}$ after 5 abration times. Mechanical stability of the $\mathrm{GSF}_{300}$ was also evaluated via tape peeling. As shown in Fig. $5 \mathrm{~b}$, repeated peeling with the new tape results in gradual decrease of the contact angle, and
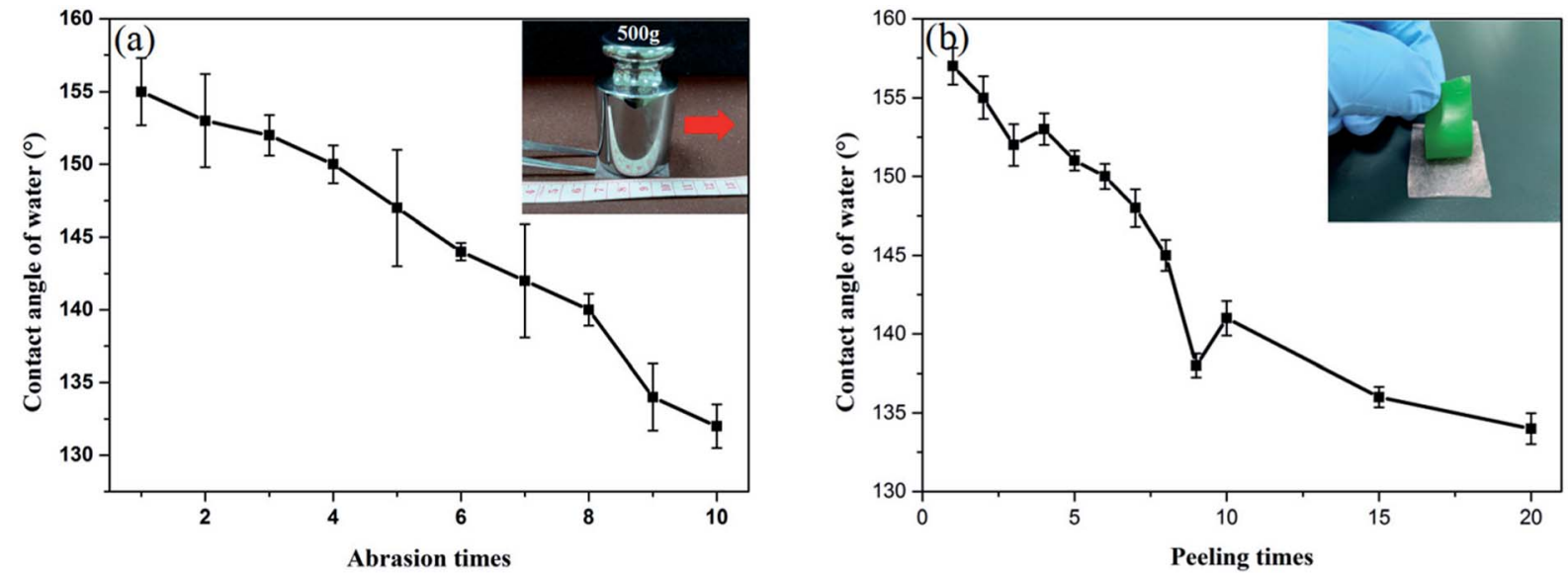

Fig. 5 (a) Variation of WCA of the GSF 300 with peeling times in the tape peeling test. The inset shows schematic of the tape peeling test. (b) Variation of WCA of the GSF 300 with abrasion times in the abrasion test. The inset shows schematic of the sandpaper abrasion test. 


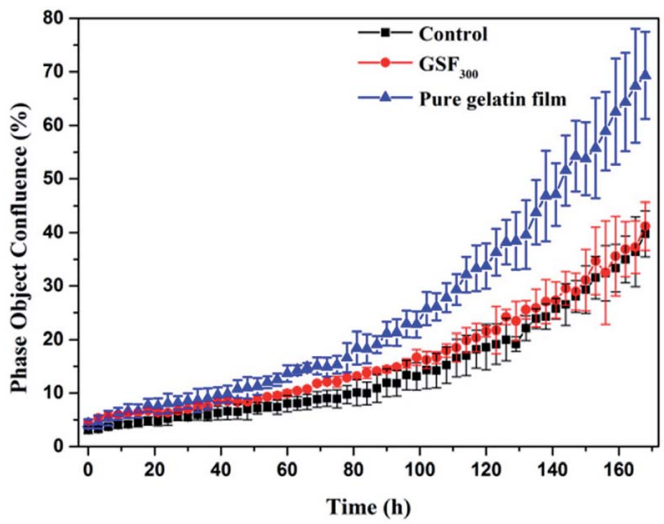

Fig. 6 Real-time cell confluence study in human fibroblast cell lines (hFOB 1.19). The cell population was monitored for $168 \mathrm{~h}$ using an Incucyte ${ }^{\mathrm{TM}} \mathrm{Zoom}$ system in an incubator $\left(5 \% \mathrm{CO}_{2}\right.$ and $\left.37^{\circ} \mathrm{C}\right)$

Table 3 The GRG and cytotoxicity level of the $\mathrm{GSF}_{300}$ and pure gelatin film

\begin{tabular}{llllll}
\hline & & 1 day & 3 day & 5 day & 7 day \\
\hline GSF $_{300}$ & GRG (\%) & 122 & 133 & 116 & 104 \\
& Cytotoxicity level & 0 & 0 & 0 & 0 \\
Pure gelatin & GRG (\%) & 156 & 164 & 181 & 174 \\
film & Cytotoxicity level & 0 & 0 & 0 & 0
\end{tabular}

after peeling for 5 times, the film is still superhydrophobic. From the above tests, it demonstrates that the obtained $\mathrm{GSF}_{300}$ has good mechanical stability.

As potential implantable materials, we hope that the obtained superhydrophobic gelatin films need to have good biocompatibility and nontoxicity. Therefore, the proliferation of $\mathrm{GSF}_{300}$ and pure gelatin film were studied by real-time monitoring of human fibroblast cell lines (hFOB 1.19) using an Incucyte ${ }^{\mathrm{TM}}$ Zoom microscope. As shown in Fig. 6, timelapse imaging of the blank control shows a standard growth curve up to $39 \%$ cell confluence level by day 7 , while the pure gelatin film reaches $69 \%$ cell confluence level by day 7 . It indicates that the pure gelatin film efficiently promotes the cell proliferation. Meanwhile, the growth curve of $\mathrm{GSF}_{300}$ was a little higher than the blank control, which represents that the $\mathrm{GSF}_{300}$ has certain promoting effect on cell proliferation. The cytotoxicity was expressed by the relative growth rate (RGR, \%) as follow in Table 3 . It is found that all the relative growth rates are more than $100 \%$, corresponding to the cytotoxicity level of 0 per the standard (Table S1, ESI $\dagger$ ). In addition, as shown in Fig. 7, the human fibroblasts cells cultured in extraction media from the pure gelatin film and $\mathrm{GSF}_{300}$ are marked by a green phase object mask via the software of Incucyte $^{\mathrm{TM}}$ Zoom, which display healthy spindle-like or starlike shape, and the amount of cells rised with the extension of culture time, suggesting that $\mathrm{GSF}_{300}$ imposes no suppression on the growth of cells.

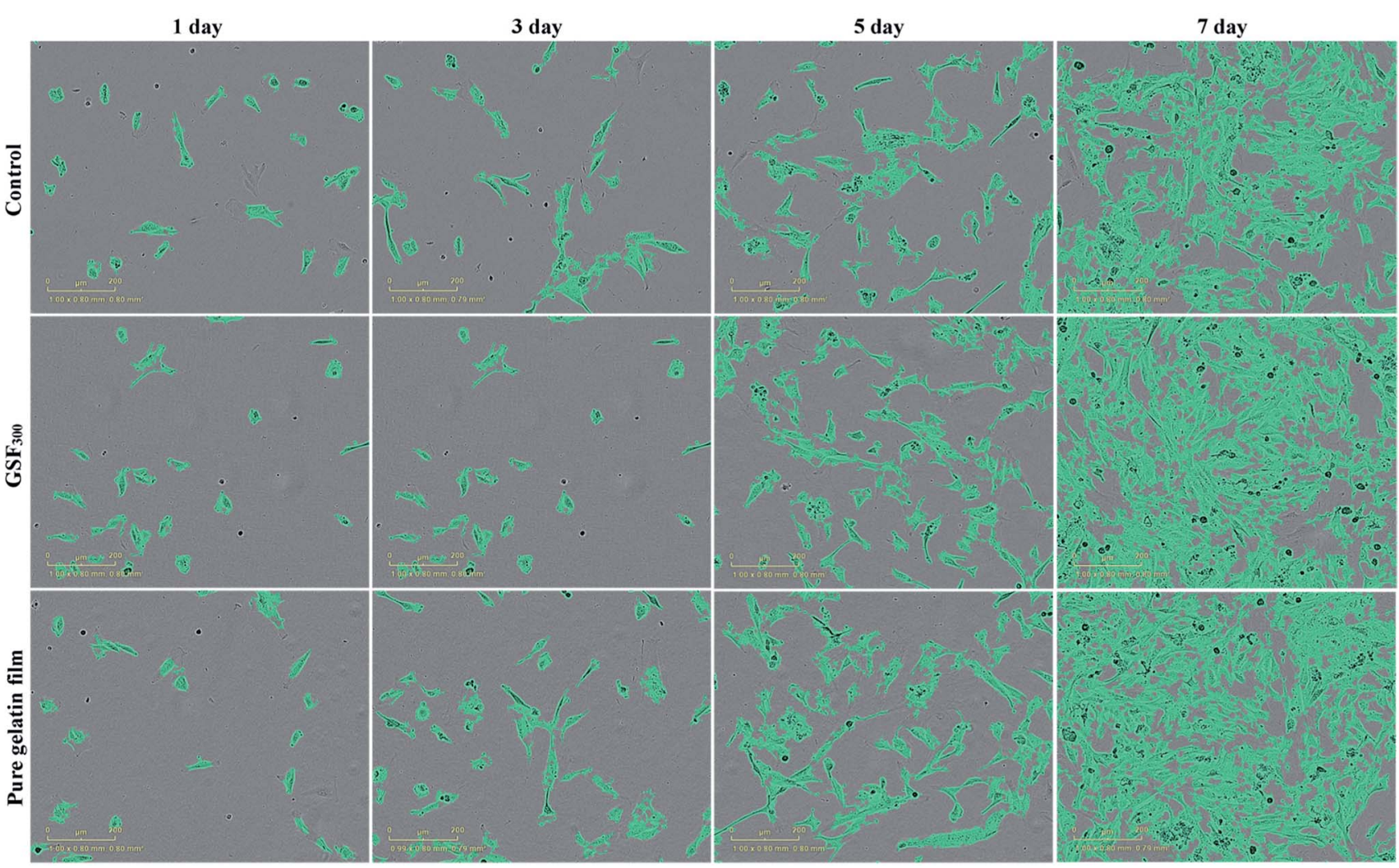

Fig. 7 Cell morphology of GSF 300 and pure gelatin film at point of 1, 3, 5, 7 day. Scale bar: $200 \mu \mathrm{m}$. 


\section{Conclusions}

In summary, we have prepared a novel superhydrophobic gelatin films by using the silica nanoparticles and further functionalized by FAS molecules as superhydrophobic coating. Formaldehyde and glycerol were added into the gelatin film as a crosslinker and plasticizer respectively to improve the mechanical properties. We have observed that the hydrophobic ability was strongly related to the size of modified particles. The superhydrophobic film $\left(\mathrm{GSF}_{300}\right)$ modified by silicon nanoparticles with particle size of $303 \mathrm{~nm}$ has the largest contact angle. What's more, $\mathrm{GSF}_{300}$ has good mechanical strength, durability and nontoxicity. Therefore, such a durable, robust, superhydrophobic film may be useful for developing for various functional biomedical applications.

\section{Conflicts of interest}

There are no conflicts to declare.

\section{Acknowledgements}

This work was supported by State Natural Sciences Fund, China (Project No. 21506236, 51372276)

\section{References}

1 L. L. Wang, D. Chen, K. Jiang and G. Z. Shen, Chem. Soc. Rev., 2017, 46, 6764-6815.

2 C. Shi, C. Zhuang, Y. Cui and F. Tao, Polym. Bull., 2017, 74, 121.

3 J. J. Xue, R. Shi, Y. Z. Niu, M. Gong, P. Coates, A. Crawford, D. F. Chen, W. Tian and L. Q. Zhang, Colloids Surf., B, 2015, 135, 846-854.

4 P. Sánchez, J. L. Pedraz and G. Orive, Int. J. Biol. Macromol., 2017, 98, 486.

5 S. D. Kim, S. L. Hong, M. J. Kim, J. Y. Kim, Y. W. Kim, S. K. Koo and K. S. Cho, Auris, Nasus, Larynx, 2018, 45, 286-290.

6 Y. Zhang, Q. Zhao, H. Wang, X. Jiang and R. Cha, Carbohydr. Polym., 2017, 164, 358-363.

7 S. Afshar and H. Banisadi, Int. J. Biol. Macromol., 2018, 109, 1019-1028.

8 C. G. Shi, F. R. Tao and Y. Z. Cui, Int. J. Biol. Macromol., 2018, 109, 863-871.

9 L. L. Deng, X. Zhang, Y. Li, F. Que, X. F. Kang, Y. Y. Liu, F. Q. Feng and H. Zhang, Food Hydrocolloids, 2017, 75, 72-80.

10 F. Guo and Z. G. Guo, RSC Adv., 2016, 6, 36623-36641.

11 S. Yu, Z. G. Guo and W. M. Liu, Chem. Commun., 2015, 51, 1775-1794.
12 G. Wen, Z. Guo and W. Liu, Nanoscale, 2017, 9, 3338-3366.

13 J. L. Yong, F. Chen, Q. Yang, J. L. Huo and X. Hou, Chem. Soc. Rev., 2017, 46, 4168-4217.

14 Z. Q. Yang, L. D. Wang, W. Sun, S. J. Li, T. Z. Zhu, W. Liu and G. C. Liu, Appl. Surf. Sci., 2017, 401, 146-155.

15 C. L. Xu, F. Song, X. L. Wang and Y. Z. Wang, Chem. Eng. J., 2017, 313, 1328-1334.

16 J. L. Yong, F. Chen, Q. Yang, J. L. Huo and X. Hou, Chem. Soc. Rev., 2017, 46, 4168-4217.

17 Y. H. Wu, W. J. Zhao, W. R. Wang and W. J. Sui, RSC Adv., 2016, 6, 5100-5110.

18 M. Z. Ge, C. Y. Cao, J. Y. Huang, X. N. Zhang, Y. X. Tang, X. R. Zhou, K. Q. Zhang, Z. Chen and Y. K. Lai, Nanoscale Horiz., 2018, 3, 235-260.

19 H. Zhu, Z. G. Guo and W. M. Liu, Chem. Commun., 2014, 50, 3900-3913.

20 R. Yang, A. Asatekin and K. K. Gleason, Soft Matter, 2012, 8, 31-43.

21 Y. C. Fu, J. X. Jiang, Q. H. Zhang, X. L. Zhan and F. Q. Chen, J. Mater. Chem. A, 2017, 5, 275-284.

22 S. T. Yohe, Y. L. Colson and M. W. Grinstaff, J. Am. Chem. Soc., 2012, 134, 2016-2019.

23 S. T. Yohe, J. A. Kopechek, T. M. Porter, Y. L. Colson and M. W. Grinstaff, Adv. Healthcare Mater., 2013, 2, 1204-1208.

24 W. Stöber, A. Fink and E. Bohn, J. Colloid Interface Sci., 1968, 26, 62-69.

25 J. Zhao, F. Pan, P. Li, C. Zhao, Z. Jiang, P. Zhang and X. Cao, ACS Appl. Mater. Interfaces, 2013, 5, 13275-13283.

26 K. Pietrucha and M. Safandowska, Process Biochem., 2015, 50, 2105-2111.

27 M. S. Hoque, S. Benjakul and T. Prodpran, Food Hydrocolloids, 2011, 25, 82-90.

28 P. Bergo and P. J. A. Sobral, Food Hydrocolloids, 2007, 21, 1285-1289.

29 Y. L. Li, C. Liu, Y. Tan, K. Xu, C. G. Lu and P. X. Wang, Carbohydr. Polym., 2014, 110, 87.

30 W. Treesuppharat, P. Rojanapanthu, C. Siangsanoh, H. Manuspiya and S. Ummartyotin, Biotechnol. Rep., 2017, 15, 84 .

31 J. D. Brassard, D. K. Sarkar and J. Perron, ACS Appl. Mater. Interfaces, 2011, 3, 3583-3588.

32 R. Arora and W. Mosch, J. Mater. Chem., 2012, 22, 5784-5791. 33 L. Shi, Q. Yu, Y. Y. Mao, H. B. Huang, H. W. Huang, Z. Z. Ye and X. S. Peng, J. Mater. Chem., 2012, 22, 21117-21124.

34 S. Rivero, M. A. García and A. Pinotti, Innovative Food Sci. Emerging Technol., 2010, 11, 369-375.

35 S. Sanwlani, P. Kumar and H. B. Bohidar, J. Phys. Chem. B, 2011, 115, 7332-7340. 